\title{
RF-THERMAL-STRUCTURAL ANALYSIS OF A WAVEGUIDE HIGHER ORDER MODE ABSORBER*
}

\author{
G. Cheng", E. F. Daly, R. A. Rimmer, M. Stirbet, L. Vogel, H. Wang, K. M. Wilson, Jefferson Lab, \\ Newport News, VA, U.S.A.
}

\begin{abstract}
For an ongoing high current cryomodule project, a total of 5 higher order mode (HOM) absorbers are required per cavity. The load is designed to absorb Radio Frequency (RF) heat induced by HOMs in a $748.5 \mathrm{MHz}$ cavity. Each load is targeted at a $4 \mathrm{~kW}$ dissipation capability. Water cooling is employed to remove the heat generated in ceramic tiles and by surface losses on the waveguide walls. A sequentially coupled RF-thermal-structural analysis was developed in ANSYS to optimize the HOM load design. Frequency-dependent dielectric material properties measured from samples and RF power spectrum calculated by the beam-cavity interaction codes were considered. The coupled field analysis capability of ANSYS avoided mapping of results between separate RF and thermal/structural simulation codes. For verification purposes, RF results obtained from ANSYS were compared to those from MAFIA, HFSS, and Microwave Studio. Good agreement was reached and this confirms that multiple-field coupled analysis is a desirable choice in analysis of HOM loads. Similar analysis could be performed on other particle accelerator components where distributed RF heating and surface current induced losses are inevitable.
\end{abstract}

\section{HOM ABSORBER DESIGN}

The $748.5 \mathrm{MHz}$ high order mode (HOM) absorber is based on the PEP-II HOM load design [1]. The basic design specifications are as follows:

Fundamental mode frequency: $748.5 \mathrm{MHz}$

Number of HOM loads per cavity: 5

RF power deposition per load: 4000 Watts

The distribution of RF power into multiple frequency components is done in MAFIA. For each of the five HOM loads, a total of 3525.08 Watts of theoretical RF power is dissipated into the HOM load. Up to $99 \%$ of such dissipated power is absorbed by lossy ceramic tiles and removed via water cooling. Figure 1 shows a spectrum of power shares in RF frequencies, according to the MAFIA $\mathrm{RF}$ analysis. The RF power design goal for this HOM load is set to be 4000 Watts.

The RF incipient power is scattered over frequencies ranging from $1.5 \mathrm{GHz}$ to $100.5 \mathrm{GHz}$. Ideally, RF power for each frequency component needs to be included while calculating the dielectric and surface losses. At least 10 elements per propagating or resonant wavelength (the

* This manuscript has been authored by Jefferson Science Associates, LLC under U.S. DOE Contract No. DE-AC05-06OR23177, and by The Office of Naval Research under contract to the Dept. of Energy. \#cheng@jlab.org wavelength in dielectric material is affected by permittivity) are required to obtain accurate electric/ magnetic field results from ANSYS finite element analysis (FEA). Hence, frequencies higher than $6 \mathrm{GHz}$ are difficult to analyze since a giant model will be resulted. In present analysis, the power shares for frequencies lower than $6 \mathrm{GHz}$ are scaled up to achieve the 4000 Watts design goal.

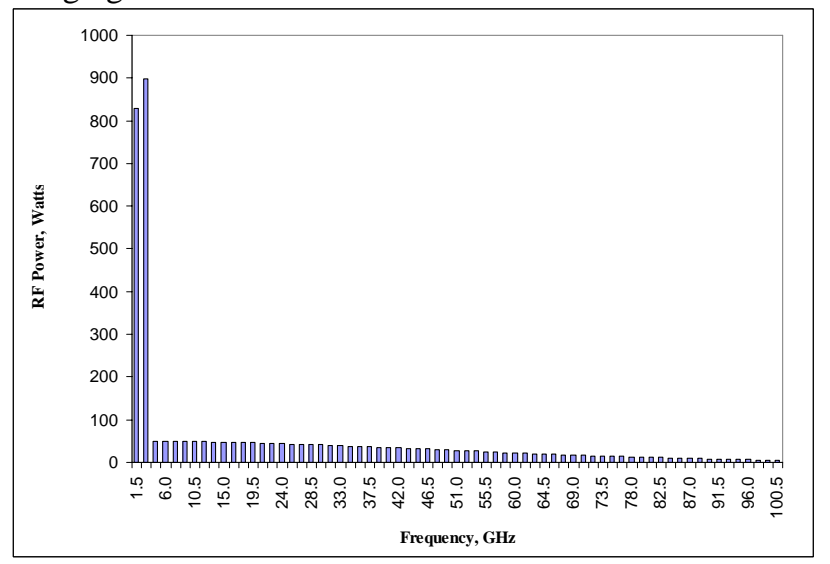

Figure 1: RF power spectrum

The ceramic tiles are cut into small pieces [1] for reduction of brazing residual stress. The standard size of one tile is $20 \mathrm{~mm} \times 20 \mathrm{~mm} \times 28.2 \mathrm{~mm}$. Some special tile shapes are used to make up an integral triangular shape. The RF design assumed a continuous ceramic tile of triangular shape. To achieve agreement with the RF design model, a pseudo-continuous tile model that uses filler material to cover up the gaps amongst tiles is employed in ANSYS RF simulation. The filler material is then assigned the same material properties as the ceramic tiles. However, the filler material is eliminated in the thermal analysis to thermally isolate the tiles from one block to another. To avoid underestimating RF heat absorbed in the filler material, another scaling factor is introduced as compensation.

Various cooling methods have been evaluated for their effectiveness in removing the $\sim 4 \mathrm{~kW}$ RF heat while maintaining structural integrity in the HOM absorber. Example concepts include a multiple loop embedded water channel, a U-shape embedded water channel, and a U-shape water cooling tube. The latter was selected at the end and all subsequent RF-thermal-structural analyses results are derived from this water cooling tube design.

\section{RF SIMULATION}

ANSYS is adopted for doing the coupled field simulation because of its multiphysics environment. Since 
the RF optimization was actually done in other software packages, the ANSYS RF results are calibrated against the RF design software. At first, the S11 magnitudes obtained from ANSYS are compared to those from MAFIA/ Microwave Studio (MWS). Table 1 exhibits the comparison.

It is seen that at lower frequencies, ANSYS agrees well with MWS on S11. At higher frequencies, the ANSYS-calculated S11's deviate a little more from MWS values. The reason is that current FEA is limited by computer memory so that the mesh size of tiles is not sufficiently fine. This situation gets worse at higher frequencies. For example, at $6 \mathrm{GHz}$, a maximum mesh size of $1.0 \mathrm{~mm}$ is required in tiles; however, a mesh size of $2.0 \mathrm{~mm}$ was actually used to obtain results presented in Table 1.

Table 1 Comparison of S11 magnitudes

\begin{tabular}{|c|c|c|c|c|}
\hline $\begin{array}{c}\text { Frequency } \\
\text { GHz }\end{array}$ & $\begin{array}{c}\text { Dielectric } \\
\text { constant }\end{array}$ & $\begin{array}{c}\text { Loss } \\
\text { tangent }\end{array}$ & $\begin{array}{c}\text { S11 } \\
\text { (MWS) }\end{array}$ & $\begin{array}{c}\text { S11 } \\
\text { (ANSYS) }\end{array}$ \\
\hline 1.497 & 24.175 & 0.2312 & 0.026 & 0.021 \\
\hline 2.994 & 22.584 & 0.1976 & 0.0441 & 0.039 \\
\hline 4.5 & 20.905 & 0.1863 & 0.0521 & 0.035 \\
\hline 6 & 21.323 & 0.1527 & 0.0438 & 0.061 \\
\hline
\end{tabular}

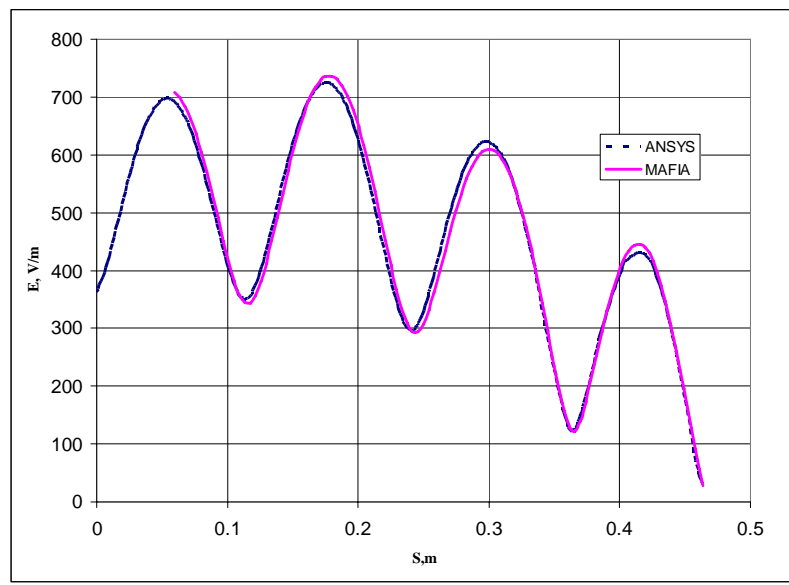

Figure 2: Comparison of electric fields in vacuum

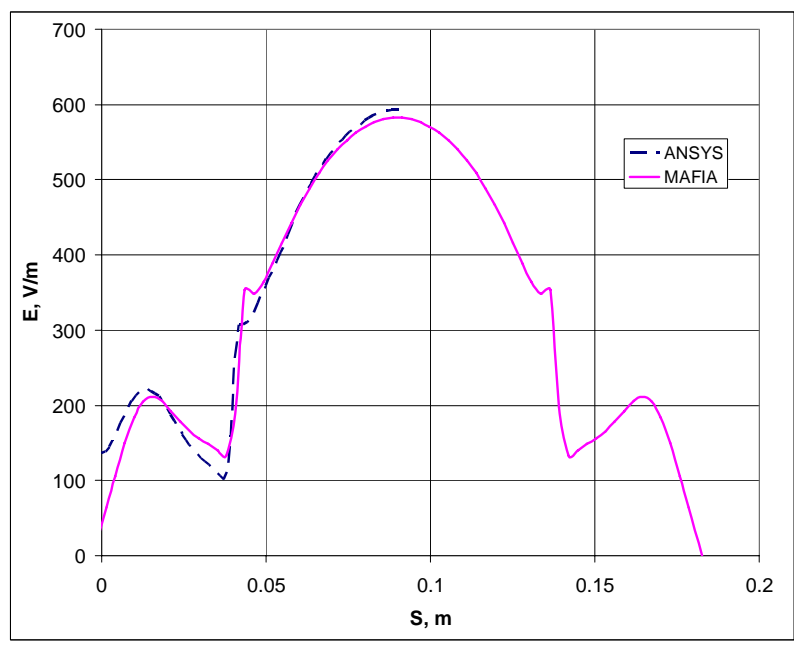

Figure 3: Comparison of electric field in tiles
The electric field distribution from ANSYS is also compared to that from MAFIA. This is because the Efield is the basis for calculation of joule heat generation in the tiles. Figures 2 and 3 show example comparisons made. A very close match in E-field magnitude was found between ANSYS and MAFIA RF simulations.

From the RF analysis, volumetric heat generation in $\mathrm{W} / \mathrm{m}^{3}$ and surface heat losses in $\mathrm{W} / \mathrm{m}^{2}$ can be evaluated. Dielectric losses are applied as heat generation in the thermal analysis and surface losses are applied as heat fluxes. The advantage of current coupled RF/Thermal analysis is that no extra efforts on mesh mapping are required. The metallic components and the RF model are meshed simultaneously at the beginning of the analysis. Thus, The vacuum meshes share common nodes with hardware meshes.

The present RF analysis considers the frequencydependent electromagnetic properties of the ceramic tiles. This means that the solutions for $1.497 \mathrm{GHz}, 2.994 \mathrm{GHz}$, $4.5 \mathrm{GHz}$, and $6 \mathrm{GHz}$ are calculated sequentially and the resultant dielectric and surface losses are superimposed in the subsequent thermal analysis.

\section{THERMAL SIMULATION}

In the thermal analysis, the joule heat generation and surface losses are transferred from the RF simulation to determine the heat transfer in the absorber waveguide. Figure 4 shows the thermal analysis model. The brazed-on U-shape OFHC cooling tube has an ID of $0.625 "$. The water flow speed, $0.81 \mathrm{~m} / \mathrm{s}$, is controlled to be $<7.5 \mathrm{ft} / \mathrm{sec}$ in order to avoid erosion in the OFHC [2]. Heat transfer coefficients for water flow in ducts are taken from literature [3] for a turbulent flow condition.

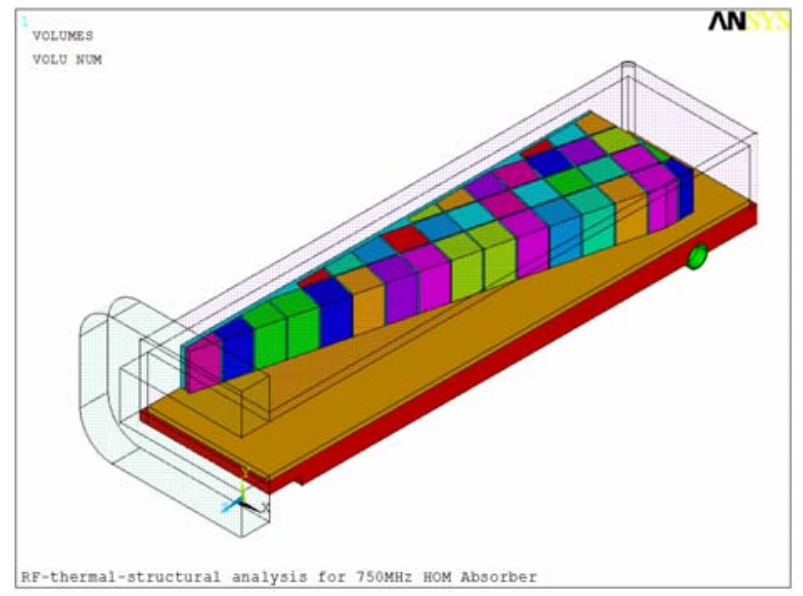

Figure 4: Thermal FEA model

To reduce the residual thermal stress during the brazing process, each ceramic tile is brazed on top of a matrix of posts [1]. Therefore, there is a mattress of needle-like posts underneath each tile triangle.

The temperature fields in the ceramic tiles and the OFHC base plate that has grooves for water cooling tube are presented in Figure 5. The highest temperature in the tiles is $410 \mathrm{~K}$. The temperatures calculated from the 
thermal analysis are loaded into the structural analysis model for the thermal stress analysis. One should bear in mind that in this particular application, it is merely temperatures that are driving structures to deform.

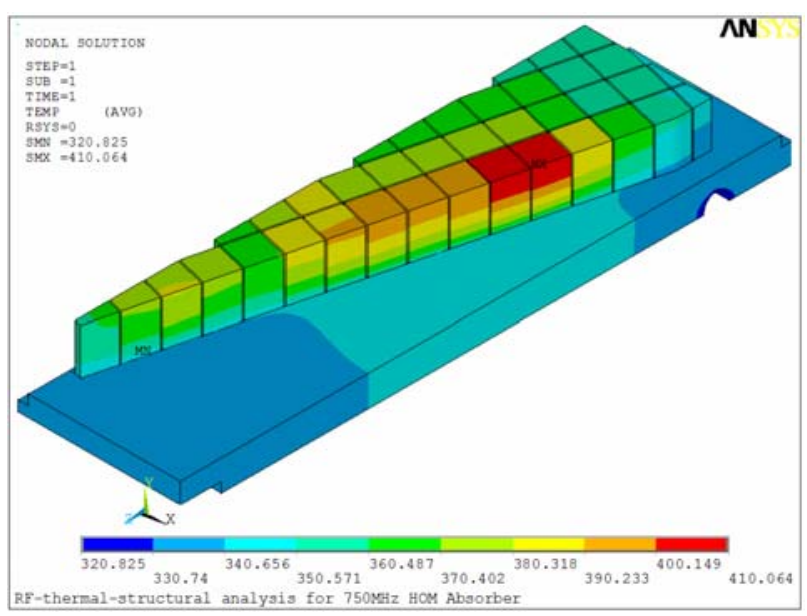

Figure 5: Temperatures in tiles and base plate

\section{STRUCTURAL STRESS ANALYSIS}

The tiles and "needle mattress" are eliminated from the structural analysis model because they are mainly followers of the thermal expansion occurring in the substrate OFHC plate. The residual stress affects local regions in the vicinity of interfacial joints between braze material and ceramic and between braze and substrate. With the present posts design concept, it is expected that the built-in residual stress will decay and diminish fast enough so that it will not influence the thermal stress developed during RF heating. The present structural analysis uses linear-elastic material properties.

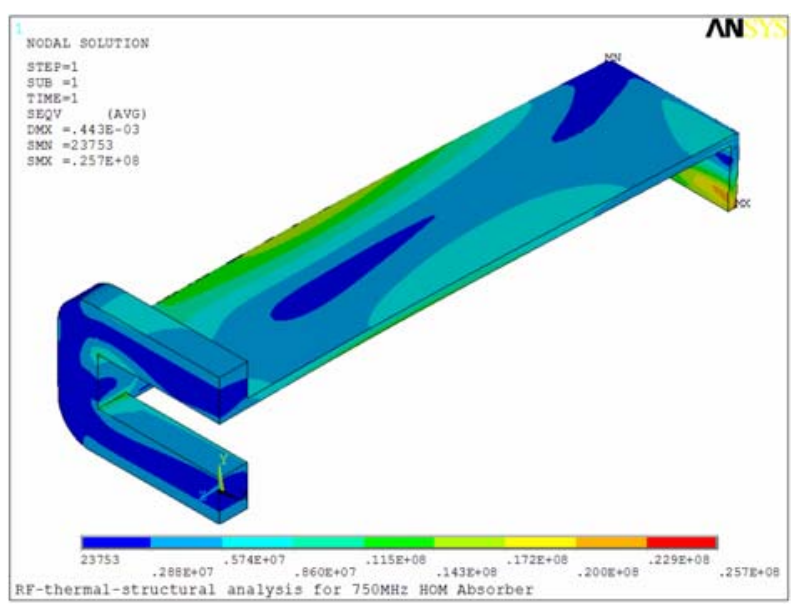

Figure 6: Von Mises Stress in stainless steel parts

The von Mises stresses in the stainless steel box and the OFHC base plate are presented in Figures 6 and 7 . Peak stress in all parts is found to be less than $40 \mathrm{MPa}$ (or $5.8 \mathrm{ksi})$. Therefore, a safety factor well above 2.0 is reached with the present cooling tube dimensions and water flow rate. It is planned that by fine tuning the cooling channel geometry and the OFHC base plate configuration, the strength of the material will be utilized to their full extent.

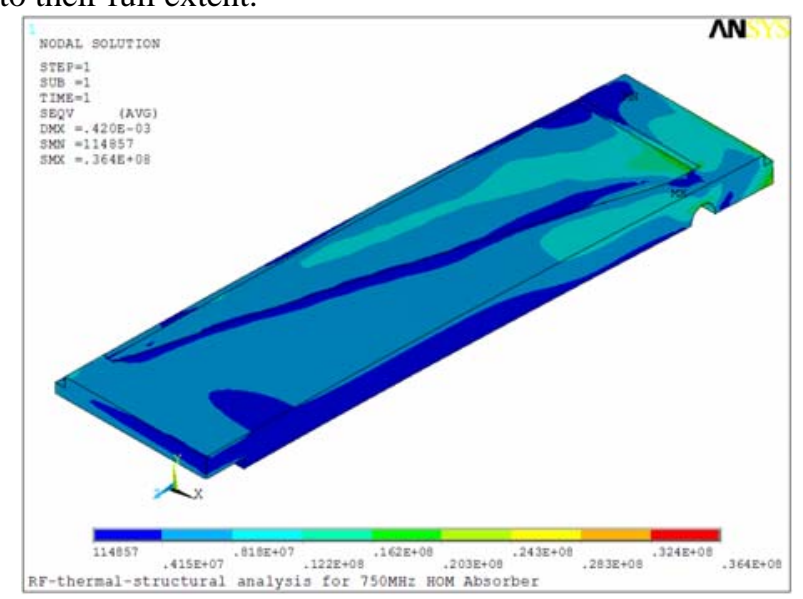

Figure 7: Von Mises Stress in OFHC parts

The robustness of the HOM waveguide under an extreme pressure load is also studied. During the longterm operation of the accelerator, it is possible that both the insulating vacuum and the beamline vacuum might be pressurized. Pressure reliefs on both the cavity and cryomodule insulating vacuum limit this pressure to less than $2.0 \mathrm{~atm}$ differential. To be conservative, a $2.0 \mathrm{~atm}$ internal/ external pressure is applied to the waveguide body to examine its capability of withstanding such a pressure load. The peak von Mises stress is found to be in the range of 64 66 MPa (9.3 9.6 ksi) and that is well below the yield strength limit of SS and OFHC.

\section{CONCLUSIONS}

The RF-thermal-structural coupled field analysis on a $748.5 \mathrm{MHz}$ HOM load has been conducted with ANSYS Multiphysics. The RF simulation results in ANSYS were compared to those from MAFIA and good agreement was reached. Thermal and structural analyses showed that the current HOM load design is robust. The approach of conducting multiple-field analysis in one software package proved to be an efficient and reliable design practice.

\section{REFERENCES}

[1] R. Pendleton, K. Ko, C. Ng, M. Neubauer, H. Schwarz, and R. Rimmer, "PEP-II B-Factory Prototype Higher Order Mode Load Design,” Proc. PAC, Dallas TX, 1995, p. 1800.

[2] K. Primdahl and R. Kustom, "Cooling the APS Storage Ring Radio-Frequency Accelerating Cavities: Thermal/Stress/Fatigue Analysis and Cavity Cooling Configuration," Proc. PAC, Dallas TX, 1995, p. 1690.

[3] General Electric Company Research and Development Center, "Heat Transfer Data Book," Section 503.3, Genium Publishing, Schenectady, NY, 1971. 